\title{
Evaluación Abierta y Transparente en Tiempo Real de Asignaturas de Ingeniería Telemática
}

\author{
Elsa Macías, Alvaro Suárez \\ Departamento de Ingeniería Telemática \\ Universidad de Las Palmas de Gran Canaria (ULPGC) \\ Edificio de Electrónica y Telecomunicación - Campus universitario de Tafira - 35017 Las Palmas de G.C. \\ elsa.macias@ulpgc.es, alvaro.suarez@ulpgc.es
}

\begin{abstract}
Resumen- Existe una cantidad enorme de métodos de evaluación de la docencia universitaria a día de hoy (tan grande como la cantidad de enseñantes). Las asignaturas de Ingeniería Telemática en España tienen distintas metodologías docentes y métodos de evaluación, pero en su mayoría tienen docencia de teoría, problemas en el aula y prácticas de laboratorio. Cada una de esas metodologías tiene sus propios métodos de evaluación particulares. Normalmente, se suelen marcar exámenes de evaluación de competencias que se realizan en una fecha determinada; elaboración de problemas o trabajos teóricos a desarrollar en un plazo prefijado; trabajos en grupo... que se evaluán después del plazo de entrega. Para este tipo de evaluación no conocemos ninguna iniciativa en la que tanto los profesores como los alumnos puedan recibir realimentación en tiempo real de la evaluación que se está realizando por parte de los profesores y comentarios de los otros alumnos. En este artículo planteamos un sistema de evaluación que permite que los alumnos y profesores puedan tener información, en tiempo real sobre la evaluación que se está realizando puntualmente. A partir de nuestra experiencia práctica, afirmamos que este sistema de evaluación ha demostrado ser muy efectivo y ahorra tiempo de revisión, a la vez que aumenta la calidad de las tareas y evaluaciones realizadas.
\end{abstract}

Palabras Clave- técnicas de evaluación individual y grupal, modelos de evaluación continua

\section{INTRODUCCIÓN}

Internet y la Web han influenciado enormemente la docencia en los últimos años. Desde el inicio de los modelos básicos de teleenseñanza, hasta los modernos modelos de enseñanza abiertos (open learning) [1] o aprendizaje sofisticado (smart learning) [2] pasando por los entornos de aprendizaje personalizados (Personalized Learning Environment (PLE)) [3] se han visto nacer, crecer y morir una gran cantidad de metodologías y plataformas diferentes. Destaca de todas estas tendencias la enseñanza abierta cuyo objetivo es que las universiades abran sus cursos a cualquier persona. En este modelo se enmarcan los cursos masivos ofertados por entidades y empresas que no son empresas nativas de educación como por ejemplo Movistar [4]. Cada uno de estos modelos y plataformas suelen llevar asociado uno o varios modelos de evaluación en los que se hacen pruebas en un momento determinado o se da un plazo de entrega de tareas y después se evalúa de forma personalizada al alumno. Por otro lado, es usual que la evaluación de los trabajos a realizar se hagan en evaluación por pares en tiempo diferido (la corrección se hace entre alumnos). Estos modelos de evaluación producen un elevado número de quejas que se producen al no existir una verificación de los trabajos que se entregan ni tampoco de una supervisión por parte de los profesores del curso.

El learning analytics [5] se suele usar como parte del modelo de aprendizaje sofisticado para recabar información del alumno. Junto con técnicas de procesado de datos masivo (Big Data) [6] puede servir para mejorar la experiencia posterior del alumno en nuevos cursos relacionados. Hasta donde alcanza nuestro conocimiento, no se suele informar al alumno ni a otros profesores del curso del conocimiento obtenido del alumno para mejorar la calidad del aprendizaje ni de la evaluación. Y mucho menos se hace esta evaluación personalizadamente ni en tiempo real. 
En este artículo presentamos una experiencia (modelo) en el marco de la evaluación del apendizaje [7] para asignaturas presenciales del área de conocimiento de Ingeniería Telemática en España, que hemos venido utilizando en los últimos años en asignaturas de master y doctorado. También se ha utilizado en cursos on line. Hasta donde alcanza nuestro conocimiento no se conoce una experiencia similar. Esta experiencia está enmarcada en nuestra trayectoria de propuestas sobre enseñanza abierta en un contexto global y no solamente abriendo los contenidos de la enseñanza a cualquier persona [8]. La idea básica es que los profesores de una asignatura evalúan de forma abierta y transparente a los alumnos (el resto de profesores puede acceder a esas evaluaciones en tiempo real); pero también los alumnos pueden acceder a esas evaluaciones. Así cualquier alumno puede mejorar su tarea en tiempo real. Este proceso se lleva a cabo dentro de un plazo marcado inicialmente por los profesores de acuerdo con los alumnos. Los resultados obtenidos son muy alentadores puesto que fomenta una elaboración de trabajos de forma competitiva (logrando una calidad superior a los trabajos que se realizan sin este tipo de evaluación), y además se logra una evaluación más completa que la tradicional y de mayor calidad fomentando la competitividad sana entre los alumnos y profesores. Aunque el tiempo invertido en la evaluación por parte de los profesores es mayor que en la tradicional; este método de evaluación permite un ahorro de tiempo considerable en la fase de revisión de las evaluaciones que se reduce a cero prácticamente.

La estructura de este artículo es la siguiente: en la sección II se presenta el marco de aplicación de este nuevo método de evaluación, la idea básica, sus ventajas e inconvenientes. En la sección III se presenta la aplicación de este método a asignaturas de nuestro entorno académico y en la sección IV se presentan las principales conclusiones.

\section{EVALUACIÓN DEL APRENDIZAJE ABIERTA, TRANSPARENTE Y EN TIEMPO REAL}

En este apartado presentamos, en primer lugar el contexto de aplicación de nuestro método, después revisamos brevemente las ideas básicas del modelo de evaluación tradicional, a continuación revisamos brevemente las ideas principales de nuestro nuevo modelo de evaluación del aprendizaje.

\section{A. Metodologías docentes de las asignaturas de Ingeniería Telemática}

En [9] se presentan algunas metodologías adaptadas al Espacio de Educación Superior Europeo. Muchas universidades en España, basándose en este trabajo han elaborado un catálogo de metodologías docentes y de evaluación para sus estudios. En el caso de la Universidad de Las Palmas de Gran Canaria
$(U L P G C)$, se estipula qué metodologías docentes y evaluación puede llevarse a cabo en sus estudios [10]. Las metodologías docentes recogidas en este Reglamento que se suelen aplicar a las materias de Ingeniería Telemática se recogen en los documentos VERIFICA de los títulos que se imparten en la Escuela de Ingeniería de Telecomunicación y Electrónica (EITE) [11], que por comodidad se resumen brevemente:

a) Modalidades de enseñanza presencial:

- Teórica: clase teórica, seminario, tallertrabajo en grupo, clase teórica de problemas o casos, evaluación.

- Práctica: laboratorio (grupos pequeños o medianos) y evaluación,

- Común: Tutoría (grupos pequeños o medianos).

b) Modalidades de trabajo autónomo del alumno:

- Trabajos teóricos.

- Estudio teórico.

- Trabajos prácticos.

- Estudio práctico.

- Actividades complementarias.

En cada universidad también existe reglamentación específica sobre el tipo de evaluación y actividades evaluables que se pueden llevar a cabo. En el caso de la ULPGC se especifica en el artículo 14 del Reglamento de Evaluación de los Resultados de Aprendizaje y de las Competencias adquiridas por el alumnado en los Títulos Oficiales, Títulos Propios y de Formación Continua de la ULPGC [12], el tipo de actividades evaluables, de ellas nuestro método se puede aplicar especialmente para:

a) Actividades de evaluación con soporte virtual.

b) Actividades virtuales.

c) Presentaciones o exposiciones individuales o en grupo.

d) Trabajos individuales o en grupo (el uso fraudulento del trabajo de otros como si se tratara del de uno mismo y con la intención de aprovecharlo en beneficio propio acarreará las responsabilidades previstas en el artículo $30 \mathrm{del}$ presente Reglamento).

Destacar que la valoración de los trabajos encargados al estudiante, de forma individual o grupal, estarán orientados a la comprobación de las competencias adquiridas por los estudiantes.

Nuestro método se puede aplicar tanto para evaluación individual como para evaluación en grupo.

En el artículo 18 se regula el tipo de evaluaciones posibles:

a) Pruebas o exámenes escritos. 
b) Pruebas o exámenes orales.

c) Actividades de laboratorio, clínicas o de campo, prácticas, seminarios o talleres.

d) Trabajos, para cuya ejecución será necesario la previa determinación por el profesorado de las condiciones de realización, de la exposición y de la puntuación que se otorgue, respetando lo aprobado en el proyecto docente.

e) Prácticas externas.

f) Proyecto o Trabajos Fin titulación, y

g) Otras actividades que se detallen en el Proyecto Docente.

Nuestro método no se puede aplicar para la evaluación de tipo: $b$ (porque al ser oral no hay posibilidad de realimentación durante un tiempo), $e$ (porque cada empresa trata a los alumnos de manera específica) ni $f$ (porque sólo hay cooperación entre el tutor y el alumno generalmente).

Finalmente subrayar que el mencionado Reglamento prevé que:

a) El estudiante tiene derecho a solicitar sus resultados en toda prueba, trabajo o examen realizado, de acuerdo con el sistema de evaluación previamente establecido en el proyecto docente de la asignatura.

b) Los trabajos y memorias de prácticas, una vez. calificados, se devolverán a los interesados siempre que lo soliciten por escrito.

c) Junto con los resultados de las evaluaciones, los profesores deberán hacer público el horario, el lugar y la fecha en que tendrá lugar su revisión, con un mínimo de dos sesiones que no podrán coincidir en el mismo día, y

d) Las calificaciones finales pueden ser objeto de reclamación por los estudiantes.

Uno de los objetivos de nuestro método es precisamene ahorrar tiempo del profesor y el alumno en estos menesteres.

\section{B. El método de evaluación tradicional}

En la Fig. 1 se esquematiza el proceso tradicional de evaluación de actividades:

a) El profesor coordinador (de acuerdo con el Proyecto Docente de la asignatura), avisa a los alumnos que tienen un plazo determinado para realizar una actividad docente, establece los requisitos para realizarla, recuerda los criterios de evaluación y marca las pautas para el qué y cómo se debe hacer. b) El alumno realiza la actividad y la envía a su profesor para que éste la evalúe. Normalmente el alumno suele procastinar [13] y la envía en el límite del plazo, aunque existan aplicaciones móviles para evitarlo [14].

c) El profesor evalúa las actividades de los alumnos (individualmente si la actividad es individual) o a cada grupo por separado (si la actividad es grupal). Normalmente, en nuestra experiencia, los alumnos no acceden a las actividades del resto (incluso habiendo trabajado en grupo) porque se reparten las tareas y las hacen de forma independiente, $\mathrm{y}$ procastinan $\mathrm{y}$ entregan las actividades justo en el plazo marcado.

d) El profesor informa de las notas obtenidas por cada uno de los alumnos; pero la evaluación de cada actividad sólo la conoce cada alumno por separado (o en grupo; pero no entre grupos). Algunos profesores también procastinan y cuando el alumno recibe la evaluación ya ha pasado el tiempo suficiente como para que el alumno pierda el interés en la evaluación o incluso ya ni se acuerde de los contenidos evaluados.

e) Se establece un plazo reclamaciones para respetar la reglamentación pertinente.

Este proceso lleva su tiempo y si es largo puede desmotivar al alumno en su proceso enseñanzaaprendizaje, y al profesor por verlo como una carga de trabajo desmotivante [15].

\section{Ideas básicas del nuevo método de evaluación}

El proceso de evaluación tradicional es directo, en una sola pasada, no permite realimentación directa al alumno y tampoco colectiva.

El modelo de manejo de procesos que nos ha inducido a emplear nuestro nuevo modelo de evaluación son las técnicas ágiles de desarrollo de proyectos [16]. Porque por un lado, a día de hoy el desarrollo de proyectos se lleva a cabo con participación directa de los clientes (en nuestro caso los profesores), atendiendo a los cambios (razonables) de análisis de requisitos que hacen los clientes y aceptan los desarrolladores (alumnos en nuestro caso). Por otro lado, esta técnica supone un entrenamiento para el desarrollo profesional del futuro ingeniero o doctor.

El objetivo principal es que toda la información de evaluación (y el proceso de evaluación en sí mismo) fluya de manera transparente y en tiempo real entre profesores y alumnos y se minimicen las reclamaciones finales de los alumnos.

El método que hemos venido usando en los dos últimos años consiste en (Fig. 2): 


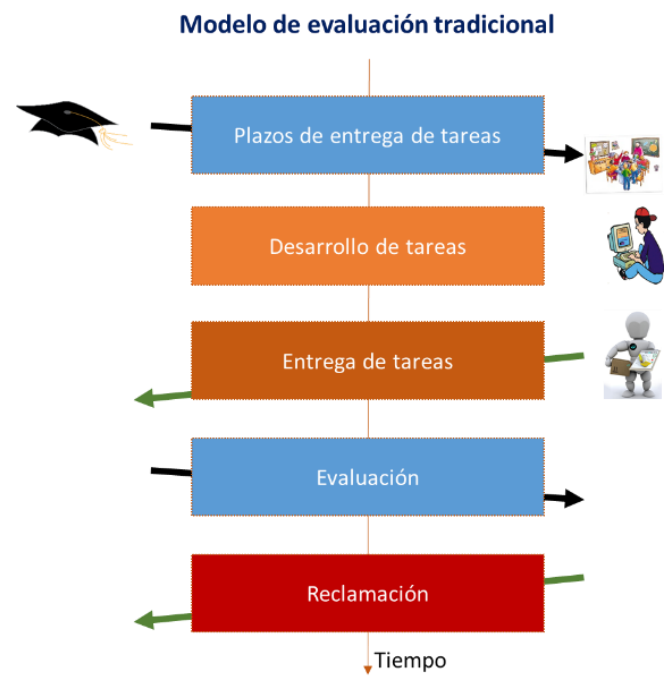

Fig. 1. Método tradicional de evaluación al final del plazo de entrega de tareas marcadas por el profesor

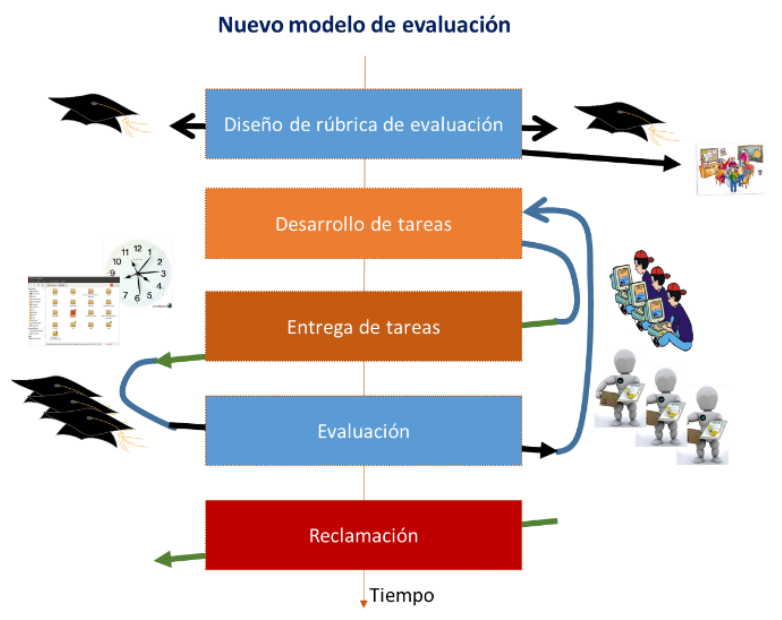

Fig. 2. Nuevo método de evaluación durante el plazo de entrega de tareas marcadas por el profesor

a) Los profesores de la asignatura establecen una rúbrica [17] para establecer criterios comunes de corrección y que se da a conocer a los alumnos.

b) Se lleva a cabo la fase (a) del proceso tradicional, con una variación: los profesores evalúan la actividad cada vez que detecten cambios significativos (que incluyan cierta envergadura a considerar por parte del profesor), o a petición del alumno o simplemente una vez cada cierto número de días. El objetivo es que no haya una única evaluación al finalizar el plazo de entrega.

c) Las actividades se depositan en un lugar conocido por todos los alumnos (por ejemplo una carpeta compartida en La Nube si se trata de la memoria escrita o software), y todos los profesores. d) Se implanta un sistema de versiones para controlar las correcciones que han ido haciendo cada uno de los profesores (visibles al resto de profesores y a todos los alumnos). De esta manera cada alumno puede recibir sugerencias o ayudas por parte de otros alumnos, para que mejore sus trabajos en función de las correcciones que observa del profesor y su punto de vista de cómo mejorar la actividad. La calificación final premiará a aquellos alumnos que realicen sugerencias o ayudas a los demás.

e) Se implanta un registro personalizado de mejoras realizadas por el alumno en respuesta a las correcciones de los profesores y a comentarios de sus compañeros. Este registro debe estar anotado con valores de tiempo que indiquen cuando se han hecho esas mejoras.

f) En base a esas anotaciones de tiempo se premiará a quellos alumnos que hayan realizado las mejoras antes que otros y también a los que hayan aconsejado mejoras (en especial a aquellos que hayan aportado información de revistas, libros o páginas Web que ayuden a mejorar la actividad). También se hace pública la información relativa a cuando los profesores han hecho sus correcciones y por supuesto qué correcciones han propuesto.

De esta manera se evita la procastinación de los alumnos, porque de manera implícita, aquel que no realice mejoras rápidamente va a ir viendo reducida su capacidad de superar la evaluación al ir obteniendo notas muy bajas (apartado e). También logra que el profesor realice de forma rápida sus evaluaciones para que los alumnos tengan la mayor probabilidad de obtener buena nota y mantener su "buen hacer" frente al resto de profesores y alumnos (que le evalúan en las encuestas oficiales). Esto es, se fomenta una competencia sana entre profesores y entre alumnos.

También se permite mejorar la calidad de las actividades realizadas por los alumnos puesto que todos pueden ver las que realiza el resto. $\mathrm{Y}$ se fomenta que los alumnos cooperen entre ellos porque reciben mejor calificación si se ayudan entre ellos (apartado d). Un efecto colateral es que todos los alumnos tienen información de las actividades realizadas por todos, y no sólo de la que ellos han realizado.

Se consigue una normalización de la forma de corregir que no es posible conseguir con una rúbrica que sólo indica qué y cómo se debe evaluar; pero no permite observar cuál es la evaluación realizada. Además, todos los profesores pueden observar en tiempo real cuales son las correcciones que están haciendo el resto de profesores así como los comentarios de alumnos. De esta forma se puede minimizar la cantidad de reclamaciones que se suelen recibir por parte de los alumnos. Además, como los alumnos han tenido información en tiempo real de sus 
evaluaciones pueden ir observando cómo se les ha ido corrigiendo (a todos), minimizando sospechas de que a otros se les haya tratado de forma diferente en la evaluación. Esto también minimiza las reclamaciones. En cualquier caso, si existieran reclamaciones más allá del ámbito de actuación del profesor coordinador (a la Comisión de Asesoramiento Docente o a instancias superiores), toda la información compatida se haría accesible a esas instancias para que tengan información detallada de lo ocurrido. Es decir, el sistema permite por sí mismo la auditoría detallada de la actuación de profesores y alumnos en el proceso de evaluación. Por último destacar que esta información quedaría visible a los evaluadores de las titulaciones de la Agencia Nacional de Evaluación de la Calidad y Acreditación (ANECA), como evidencias de la evaluación realizada.

Este método permite emplear técnicas de learning analytics, y aplicarla con una diferencia fundamental: todos los datos que se pueden recoger de la evaluación están disponibles a todos los profesores y alumnos en tiempo real (en realidad cualquiera de ellos podría emplear las técnicas de learning analytics porque los datos están compartidos).

El mayor inconveniente de este método es que el alumno puede verse forzado a responder de forma inmediata a las correcciones y sugerencias de mejora de sus compañeros, empleando un tiempo del que no dispondría. Por ese motivo, se aconseja relajar el criterio de tiempo de respuesta, consensuando con los alumnos los plazos de tiempo en los que se puede responder (sin ser penalizados) a las correcciones y sugerencias. Dejando libertad al alumno para que lo haga o no según su criterio y disponibilidad temporal.

Otro inconveniente importante de este método es la proporción de alumnos por profesor. Si esa proporción es elevada (más de 10) podría darse problemas para manejar los tiempos de respuesta a las actualizaciones de las actividades por parte de los alumnos.

Otro detalle importante es la labor de motivación que debe llevar a cabo el profesor para crear un ambiente de colaboración e interés por los trabajos realizados por los alumnos (entre los profesores y entre los alumnos). Sin esta motivación esta técnica es bastante difícil que de buenos resultados. Por ello se aconseja que se utlice técnicas de motivación e incluso técnicas de gamificación [18] conducentes a mejorar este aspecto parcial de la evaluación.

\section{APLICACIÓN DEL MÉTODO EN ASIGNATURAS DE INGENIERÍA TELEMÁTICA}

Nosotros utilizamos la potencia y simplicidad de Google Drive para implantar nuestro sistema. En aquellas universidades en las que es obligatorio usar las cuentas de correo electrónico para la comunicación con ellos se puede sincronizar estas cuentas para permitir el uso (a través de ellas del Google Drive). Para aquelas universidades que tengan suscrito acuerdos con otras plataformas en La Nube (Office 365 u otras) este método también es implementable. Este sistema también se puede usar en plataformas como Moodle y otros similares; pero su manejo sería bastante más tedioso tanto por parte del alumno como del profesor.

Nosotros hemos aplicado este método de evaluación en: a) La asignatura Tecnologías de Internet de Nueva Generación del Master de Ingeniería de Telecomunicación (MUIT) [19] de la EITE durante los 2 últimos cursos. b) El Seminario de Introducción a la Investigación (en los 2 últimos cursos) del Programa de doctorado Empresa, Internet y Tecnologías de las Comunicaciones (EmiTIC) [20]. c) El módulo Despliegue de redes, protocolos y servicios telemáticos en la Empresa 2.0 (en este curso) del Seminario de investigación específica del EmiTIC). En la Fig. 3 se muestran las distintas carpetas (se han puesto nombres no legibles fácilmente deliberadamente) de Google Drive compartidas para la asignatura, el seminario y el módulo en este curso académico. El uso de esta carpeta de Google Drive es muy simple tanto para el profesor como para el alumno. Configurar el acceso compartido a esas carpetas, por parte del profesor coordinador se puede llevar a cabo, de forma muy simple en minutos.

En esas carpetas, compartidas por todos los profesores y alumnos, el alumno deposita las memorias de sus tareas. Cada alumno tiene su propia carpeta (si la tarea es compleja, sino basta con un simple archivo). Cada alumno, y todos los profesores, tienen derecho a escritura en su propia carpeta y derecho a comentar en las carpetas de los otros alumnos. De esta forma se evitan posibles escrituras o modificaciones accidentales que provocan malestar entre los alumnos. Dentro de su carpeta el alumno debe ir actualizando la memoria de su tarea.

Automáticamene, Google Drive actualiza información de las modificaciones realizadas en ese archivo por parte de cualquier persona que pueda editarlo o comentarlo. Además activa marcas de tiempo de cuando se realizaron esos cambios. También permite realizar comentarios (sugerencias de los alumnos o del profesor). Esos comentarios se pueden resolver o se pueden contestar, dando lugar a conversaciones sobre cada uno de los comentarios. Un detalle importante es que Google drive permite enviar un correo electrónico por cada comentario que se haga, con lo cual se instruye a los alumnos que lo mejor es que se hagan conversaciones de comentarios para que sea posible tener un registro inmediato de cada vez que se haga un comentario en el correo electrónico de las personas que comparten el archivo. De esta manera es posible recolectar toda la información necesaria para hacer la calificación de la actividad llevada a cabo así como de 
los tiempos en los que se hicieron los comentarios y correcciones. Con el sistema de versiones de Google Drive es posible conocer exactamente la traza de actuación de cada alumno y profesor.

En la asignatura Tecnologías de Internet de Nueva Generación, obligatoria, del primer curso del MUIT, se matriculan muy pocos alumnos, y un detalle importante es que estos alumnos provienen del Grado en Ingeniería en Tecnologías de Telecomunicación que es un grado generalista con 4 intensificaciones (Sonido e Imagen, Sistemas de Telecomunicación, Electrónica e Ingeniería Telemática). En estos dos últimos años no ha cursado esta asignatura ningún alumno de Ingeniería Telemática, lo que podría llevar a pensar que los alumnos tienen menos experiencia utilizando servicios de Google. Pero en la práctica no ha sido así y los alumnos inmediatamente han dominado dichos servicios. En la Fig. 4.a se muestra el número de alumnos que ha comenzado y finalizado la asignatura en los dos últimos cursos. Se muestra que el número de alumnos es muy reducido. La cantidad de profesores que atiende a esta asignatura son dos, sin embargo el profesor encargado de las prácticas es uno sólo y el de los problemas en el aula es también uno sólo con lo cual el ratio de alumnos por profesor es reducido. La particularidad de esta asignatura en cuanto a evaluación es que al existir un solo profesor evaluador de tareas, se hace innecesario coordinar la rúbrica de evaluación.

En el Seminario de Introducción a la Investigación (EmiTIC), obligatorio, se han matriculado del orden de 20 alumnos (Fig. 4.b) y tiene una duración de 12 horas. Un detalle muy importante es que los alumnos de este seminario provienen de Ingeniería de Telecomunicación, Informática, de Arquitectura de Edificios, Ciencias Económicas y Empresariales y en menor proporción de Administración de Empresas. Es decir, la heterogeneidad del perfil de los alumnos es muy elevado. Se observa una mayor dificultad para elaborar las memorias de las tareas marcadas (básicamente un trabajo de seminario en el que deben elaborar un borrador de proyecto de investigación en su línea de investigación e innovación) entre aquellos alumnos que no son de Ingeniería de Telecomunicación ni de Informática. Sin embargo, en la práctica, todos los alumnos han podido trabajar de forma eficaz y sin mayores problemas con las carpetas compartidas y el correo electrónico para gestionar comentarios. El número de profesores del seminario es 4 y las tareas se reparten de forma equitativa entre todos ellos. Por tanto es necesario acordar una rúbrica de evaluación que incluye aspectos de la profundidad con la que se ha elaborado la memoria, la cantidad de referencias bibliográficas usadas con índice de impacto y la capacidad de síntesis de esas referencias bibliográficas, entre otros aspectos. Un parámetro importante es la cantidad de ideas que se han cruzado entre alumnos que se evalúa de forma positiva. En la práctica, la colaboración entre alumnos es muy elevada y entre ellos se cruzan mucha información proporcionándose ideas valiosas. La evaluación se va haciendo cada 3 días (el plazo es de 15 días) y en general un $60 \%$ de los alumnos suele mejorar considerablemente sus trabajos con las revisiones del profesor y comentarios de otros alumnos. Además, la homogeneización en la evaluación siempre ha sido muy elevada, dado que todos los profesores comparten sus evaluaciones.

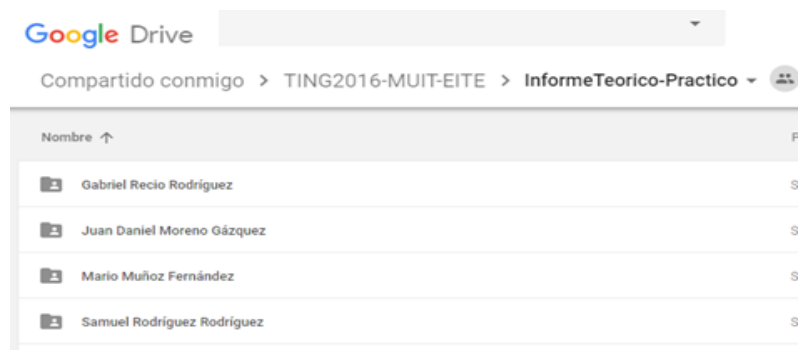

(a)

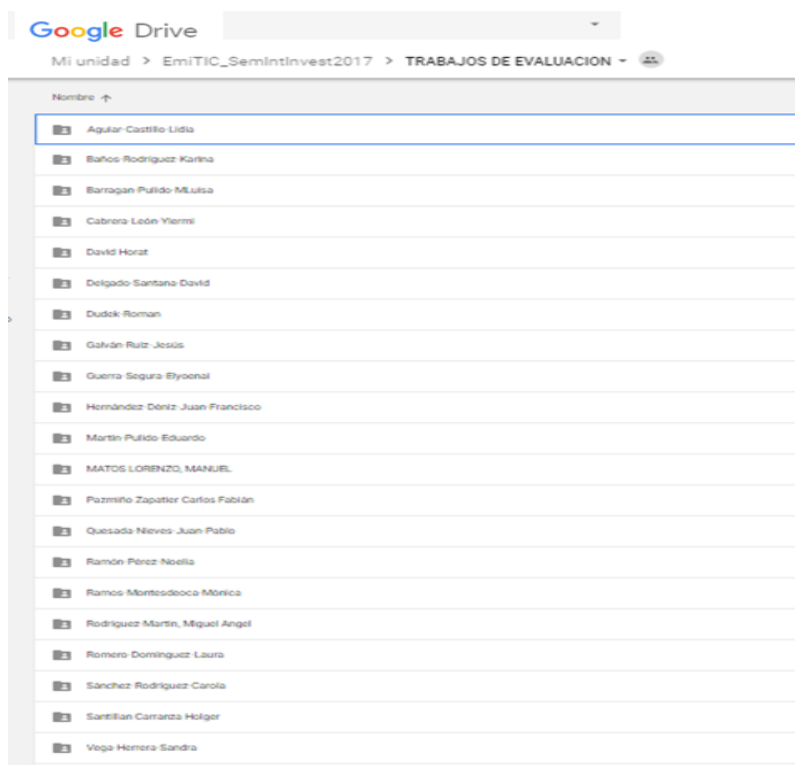

(b)

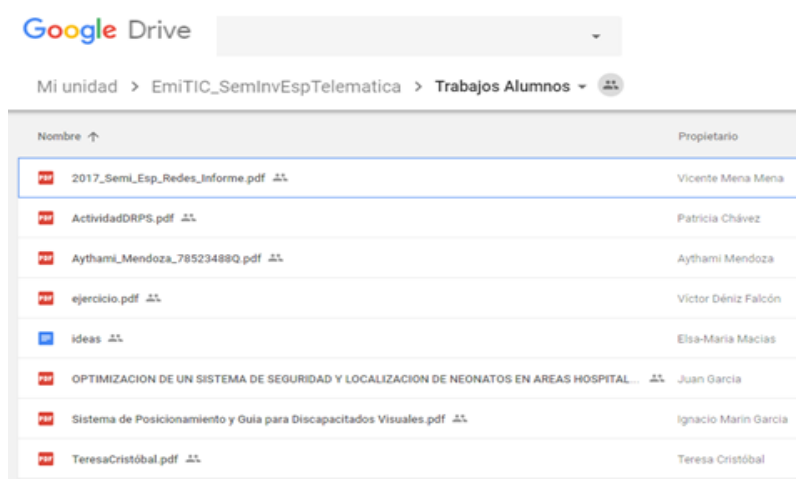

(c)

Fig. 3. Carpeta de google Drive compartidas para la asignatura, el seminario y el módulo: a) Tecnologías de Internet de Nueva Generación, b) Seminario de Introducción a la Investigación (EmiTIC) y c) Módulo Despliegue de redes, protocolos y servicios telemáticos en la Empresa 2.0 
En este curso, en el módulo Despliegue de redes, protocolos y servicios telemáticos en la Empresa 2.0 (EmiTIC), optativo, se han matriculado 7 alumnos del total de 10 que suelen asistir a las clases del segundo curso (Fig. 4.c). Todos tienen un perfil de Informática o Ingeniería de Telecomunicación, por lo que podemos decir que son homogéneos. La ventaja de este módulo es que los alumnos ya conocen bien el método de evaluación del curso anterior. En este módulo de 6 horas lo imparten tres profesores, se invita a expertos de otras universidades y la Empresa a que impartan charlas muy breves (story telling de 20 minutos) pero sólo uno de los profesores es el encargado de evaluar las memorias de los trabajos que versan sobre la optimización del despliegue de un servicio telemático innovador en la Empresa. Se pacta con los alumnos que cada 3 días, dentro del plazo de 15 días, se hacen revisiones por parte del profesor. El $60 \%$ de los alumnos suele trabajar al día a día en la mejora de sus trabajos. A los alumnos que hacen la tesis doctoral y además trabajan (alumnos a tiempo parcial), se les facilita otras temporizaciones.

En la Fig. 5 se muestran las notas obtenidas por los alumnos. El objetivo no es comparar datos cuantitativos entre distintas asignaturas (ni entre cursos d ela misma asignatura), sino demostrar que en todos los cursos, por asignatura, las notas son bastante buenas. Destacar que el tiempo dedicado a las reclamaciones de notas es nulo en todos los casos.

\section{IV.CONCLUSIONES}

La evaluación del aprendizaje del alumno es una cuestión importante en la docencia universitaria. Nosotros hemos presentado una experiencia docente en varias asignaturas con baja proporción de alumnos (menos de 10) por profesor en las que hemos aplicado nuestro método de evaluación abierta en tiempo real. La característica más importante de este método es que permite aumentar la competencia y la cooperación entre los alumnos y homogeniza el trabajo de evaluación de los profesores, minimizando la procastinación de los alumnos y reclamaciones a la calificación obtenida. Queremos destacar que en las asignaturas en las que hemos aplicado el método se ha fomentado la competitividad sana, alcanzándose en todas un grado considerable de cooperación entre profesores, entre alumnos y entre profesores y alumnos. Aunque no se ha graficado los valores, para la asignatura Tecnologías de Internet de Nueva generación, los resutlados obtenidos son mejores que en los cursos previos a los dos últimos.

Al finalizar la escritura de este artículo Google ha decidido abrir la plataforma Google Classroom (classroom.google.com) a todo aquel que tenga una cuenta activa en gmail sin necesidad de tener cuenta corporativa en Google $(G)$ Suite. Las primeras pruebas realizadas sobre dicha plataforma aconsejan migrar todo el sistema de notificación a los alumnos y manejo de carpetas compartidas y calendarios, a esta plataforma. Por tanto, como trabajo futuro pretendemos migrar toda nuestras ideas de docencia abierta a esta plataforma.

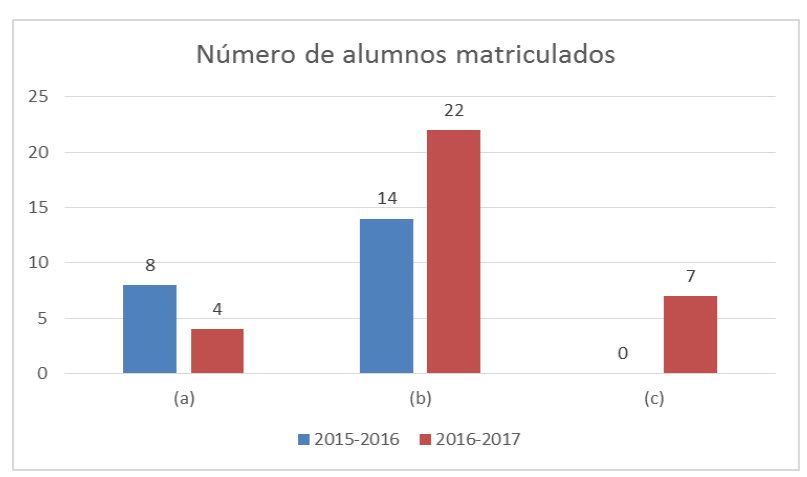

Fig. 4. Número de alumnos de los dos últimos cursos en: a) Tecnologías de Internet de Nueva Generación, b) Seminario de Introducción a la Investigación (EmiTIC) y c) Módulo Despliegue de redes, protocolos y servicios telemáticos en la Empresa 2.0 (sólo se ha impartido el último curso)
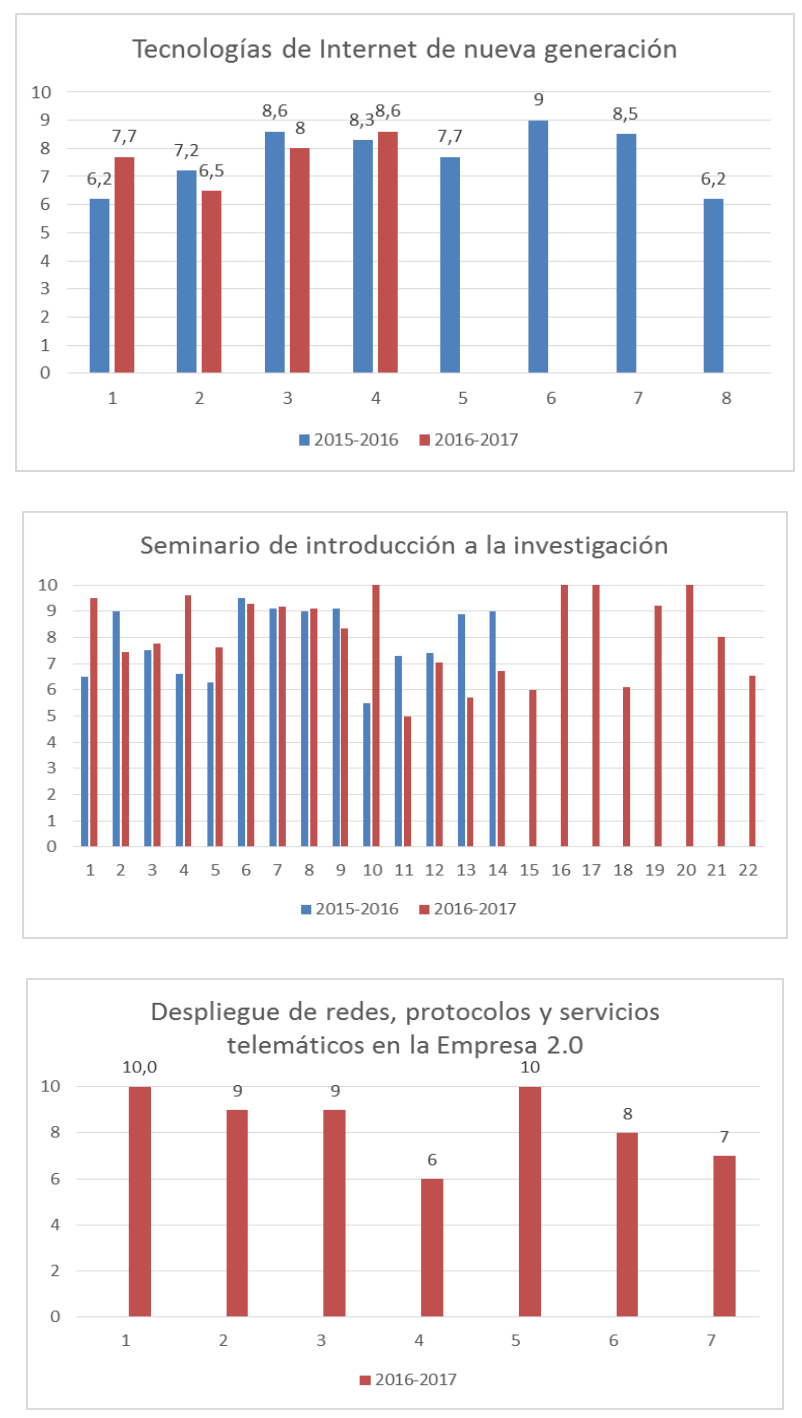

Fig. 5. Notas obtenidas por los alumnos 


\section{AGRADECIMIENTOS}

Los autores quieren agradecer a los alumnos de estas materias por haber participado de la motivación que se les trató de contagiar.

\section{REFERENCIAS}

[1] OpenLearning: [Online]. https://www.openlearning.com/

Available:

[2] SmartLearning: [Online]. Available: http://www.smartlearning.co.uk/

[3] S. Leone, Characterisation of a Personal Learning Environment as a Lifelong Learning Tool. New York: Springer-Verlag. 2013. ISBN 978-1-4614-6273-6.

[4] MiríadaX: [Online]. Available: https://miriadax.net/home

[5] M. Khalil, M. Ebner. (2016, June). What is Learning Analytics about? A Survey of Different Methods Used in 2013-2015 [Online]. https://arxiv.org/ftp/arxiv/papers/1606/1606.02878.pdf

[6] L. Joyanes, Big Data. Análisis de Grandes Volúmenes de Datos en Organizaciones. Marcombo. 2013. ISBN 978-8-4267-2081-8.

[7] B. Salinas-Fernández, C. Cotillas-Alandí (coordinadores), La evaluación de los Estudiantes en la Educación Superior. Apuntes de Buenas Prácticas. Servicio de formación permanente de la Universidad de Valencia, DL: V-4566-2007, 2007. Available:

http://www3.uji.es/ betoret/Formacion/Evaluacion/Documentaci on/La\%20evaluacion\%20estudiantes\%20en\%201a\%20ESuperior $\% 20$ UV.pdf

[8] Elsa Macías, Alvaro Suárez, "Organización de docencia cooperativa usando Google Drive ", En Actas de las III Jornadas de Innovación Educativa Telemática, 2013.

[9] M. de Miguel, "Cambio de paradigma metodológico en la Educación Superior: Exigencias que conlleva", Cuadernos de integración europea (Fundació General de la Universitat de València: Centre de Documentació Europea), ISSN 1885-1754, no. 2,2005

[10] Reglamento de planificación académica de la ULPGC, BOULPGC Año VII, nº 1, 14 de enero de 2014. [Online]. Available:

https://www2.ulpgc.es/hege/almacen/download/7107/7107474/r eglamento_de planificacion_academica.pdf
[11] Documento de Verificación del Título de Master en Ingeniería de Telecomunicación de la Escuela de Ingeniería de Telecomunicación y Electrónica (EITE). Available: http://www.eite.ulpgc.es/images/directorio_master/2015-0224\%20Documentos\%20MUIT/10.\%20Memoria\%20de\%20verifi caci\%C3\%B3n\%20del\%20t\%C3\%ADtulo_ANECA.pdf.

[12] Reglamento de Evaluación de los Resultados de Aprendizaje y de las Competencias adquiridas por el alumnado en los Títulos Oficiales, Títulos Propios y de Formación Continua de la ULPGC. Available: https://www2.ulpgc.es/hege/almacen/download/7115/7115248/1 4 enero_2014 reglamento de evaluacion.pdf.

[13] E. Herrero-Curiel, Llegan los exámenes y con ellos las ganas de procrastinar. Available: http://www.cop.es/colegiados/m13106/images/PrensaDigital3-1209.pdf.

[14] ; Deja de procrastinar! El mejor 'software' para concentrarse mientras trabajas. Available: http://www.elconfidencial.com/tecnologia/2016-05-05/softwareaplicaciones-productividad-windows-mac_1194862/.

[15] A qué dedican el tiempo los profesores universitarios, Available: http://nadaesgratis.es/admin/a-que-se-dedican-los-profesores-deuniversidad.

[16] R. C. Martin, Agile Software Development, Principles, Patterns, and Practices. Prentice Hall, 2002. ISBN: 0-13-597444-5.

[17] J. Alsina-Masmitjà (coordinador), Rúbricas Para La Evaluación De Competencias. ICE y ediciones octaedro, 2013. ISBN: 978 84-9921-476-4, $2013 . \quad$ Available: http://www.ub.edu/ice/sites/default/files/docs/qdu/26cuaderno.p df.

[18] R. S. Contreras, J. L. Eguia (Eds.), Gamificación en las Aulas Universitarias. Instituto de la Comunicación, Unversidad Autónoma de Barcelona, 2016. ISBN: 978-84-944171-6-0. Available:

http://incom.uab.cat/download/eBook incomuab_gamificacion.p df.

[19] Asignatura: Tecnologías de Internet de Nueva Generación (proyecto docente). Available: http://www2.ulpgc.es/aplicaciones/proyectosdocentes/pdf.php?id proyecto $=47946 \&$ NUEVA $=1$.

[20] Memoria de Verificación el Programa de doctorado Empresa, Internet y Tecnologías de las Comunicaciones. Available: http://edulpgc.ulpgc.es/sites/default/files/IMCE/Programas_doct orado/memorias_verificacion/Memoria\%20Definitiva\%20ANE CA\%20PD\%20EmITIC.pdf. 